\title{
Management of epidermal growth factor receptor inhibitor-associated rash: a systematic review
}

\author{
Jacqueline Brown, PhD, ${ }^{a}$ Yun Su, MD, MPH, ${ }^{b}$ Dave Nellesen, PhD, ${ }^{c}$ Pallavi Shankar, \\ $\mathrm{MPH},{ }^{\mathrm{c}}$ and Carlos Mayo, $\mathrm{MD}^{\mathrm{d}}$
}

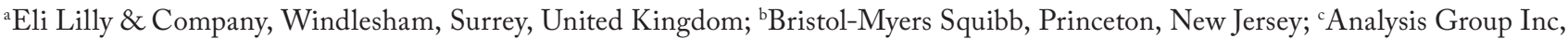
Menlo Park, California; and ${ }^{\mathrm{d} I m C l o n e ~ S y s t e m s, ~ B r i d g e w a t e r, ~ N e w ~ J e r s e y ~}$

Cancer patients treated with epidermal growth factor receptor inhibitors (EGFRIs) frequently experience skin toxicities (rash) that can compromise their quality of life and lead to dose reduction or discontinuation of treatment. Reflecting the need for effective management of EGFRI-associated rash, a number of clinical practice guidelines and management recommendations have been developed. The objective of this systematic review is to identify and summarize all available published recommendations of rash management strategies and evaluate their basis of evidence, to describe consensus in the recommendations, and where there is a lack of consensus to describe the opportunities for future clinical research to improve clinical practice in the management of EGFRI rash. Fifty-nine articles published from 2005-2011 were selected for inclusion in the systematic review. Common drug recommendations were oral and topical antibiotics, topical corticosteroids, and antihistamines; low-grade rash was generally recommended to be managed with topical antibiotics or corticosteroids, grade 2 rash with oral antibiotics or antihistamines, and severe grades of rash with oral corticosteroids or delay/dose reduction of EGFRI. The focus of clinical practice guidelines and recommendations was on reactive management. A better understanding of pre-emptive versus reactive treatment with the implementation of appropriately designed randomized controlled studies could support a more effective management of EGFRI-associated rash and improve patient outcomes. Consideration of patients' self-reported outcomes and consistent grading of rash toxicity are also recommended. Funding/sponsor Eli Lilly \& Co, Bristol-Myers Squibb

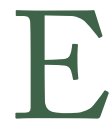
pidermal growth factor receptor inhibitors (EGFRIs) are targeted agents that are widely used to treat a range of tumor types including non-small-cell lung cancer, colorectal cancer, pancreatic cancer, and head and neck cancers. Treatment-emergent adverse events associated with EGFRI use include characteristic skin toxicities and affect roughly half of all patients treated with EGFRIs, with around $10 \%$ or more of these patients experiencing serious events of grade 3 or above. The precise incidence and severity of rash are difficult to ascertain, in part because the terminology used to describe EGFRI-associated rash varies widely. ${ }^{1-3}$

EGFRI-associated rash can severely compromise patient quality of life, resulting in substantial physical discomfort and limitation in daily activities and independence. ${ }^{4-9}$ The appearance of rash may also be associated with profound psychological impact, ${ }^{10}$ and in the context of controlled clinical studies, these effects have led to dose reduction ${ }^{4,11-13}$ or discontinu- ation of treatment. ${ }^{14-25}$ Currently marketed EGFRI treatments also include specific guidance for therapy interruption or dosage adjustment in case of rash or other dermatologic toxicity. ${ }^{26-29}$ Effective rash management therefore seems necessary to realize the benefit of EGFRI treatment and to minimize negative effects on patient quality of life.

To the knowledge of the authors, there are no published studies that comprehensively assess the consistency of rash management recommendations. The objective of this systematic review is to identify and summarize all available published recommendations of rash management strategies and evaluate their basis of evidence. Where possible, this review seeks to describe any consensus in rash management recommendations, and where there is a lack of consensus, to describe opportunities for future clinical research with the potential to improve clinical practice in the management of EGFRI rash and ultimately to improve patient outcomes.

Accepted for publication July 14, 2015. Correspondence: Dave Nellesen, PhD; Dave.Nellesen@analysisgroup.com. Disclosures: Dr Brown is employed by Eli Lilly; Dr Su, by Bristol-Myers Squibb; and Dr Mayo, by ImClone. JCSO 2016;14:21-28. @2016 Frontline Medical Communications. DOI 10.12788/icso.0193. 


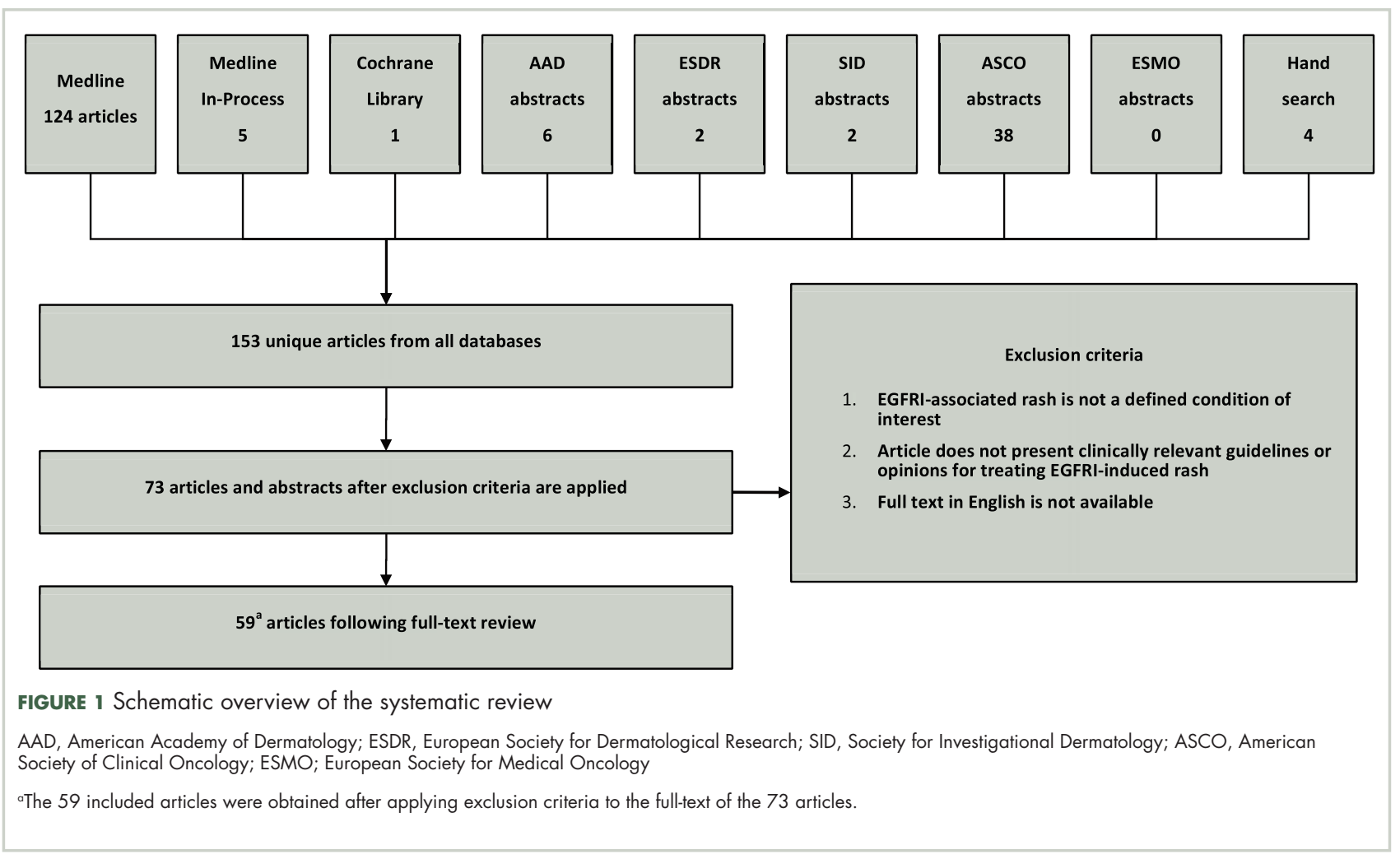

\section{Methods}

Data sources and searches

Searches were performed in June 2011 in several publication and congress databases (Figure 1). The searches were limited to articles published from 2005-2011 in the Medline, Medline In-Process, Cochrane Library, American Academy of Dermatology, Society for Investigative Dermatology, and American Society of Clinical Oncology databases. Searches in the European Society for Dermatological Research database included abstracts published from 2005-2010 (the most recent available). Searches in the European Society for Medical Oncology abstract collection was done by hand and included articles published from 2002-2010 (the most recent available). In addition, 4 articles referenced in selected articles that were deemed to be relevant but were not identified in any of the database searches were added by hand.

\section{Selection of relevant articles}

To be included in the review, the articles were required to fulfill 3 criteria:

- EGFRI-associated rash was the defined condition of interest;

- Clinically relevant guidelines or opinions for treating

EGFRI-associated rash were presented; and

- The full text in English was available.

Results included diverse article types, including reviews, case reports, and both prospective and retrospective studies (Figure 1, Table 1; Online Table 1-keywords). ${ }^{30}$

\section{Data extraction, quality assessment, and analysis}

Articles were classified according to article type, region/ perspective, rash management strategies, grade, EGFRI treatment or treatments, and evidentiary basis for conclusion. The study description/design, results, and authors' conclusions were also noted. Articles of primary research were mutually exclusively classified either as a case report, a prospective study (not a randomized controlled trial), a retrospective study, a randomized controlled trial (RTC) or a cross-sectional survey. Clinical practice guidelines were described as articles that contained systematically developed recommendations or management strategies produced under the auspices of medical specialty associations, relevant professional societies, government agencies or health care organizations or plans. If an article fitted the description of a clinical practice guideline, then it was not counted as a review article (Online Table, characteristics for all 59 articles). All data were separately extracted by 2 of the authors (PS, KY) and differences were adjudicated. Descriptive analysis using counts of article characteristics, which included study type, cancer type, and region of focus, and management strategies was performed using Microsoft Excel.

\section{Results}

A total of 153 articles were identified by the search strategy. The full text of 73 articles was reviewed, and after apply- 


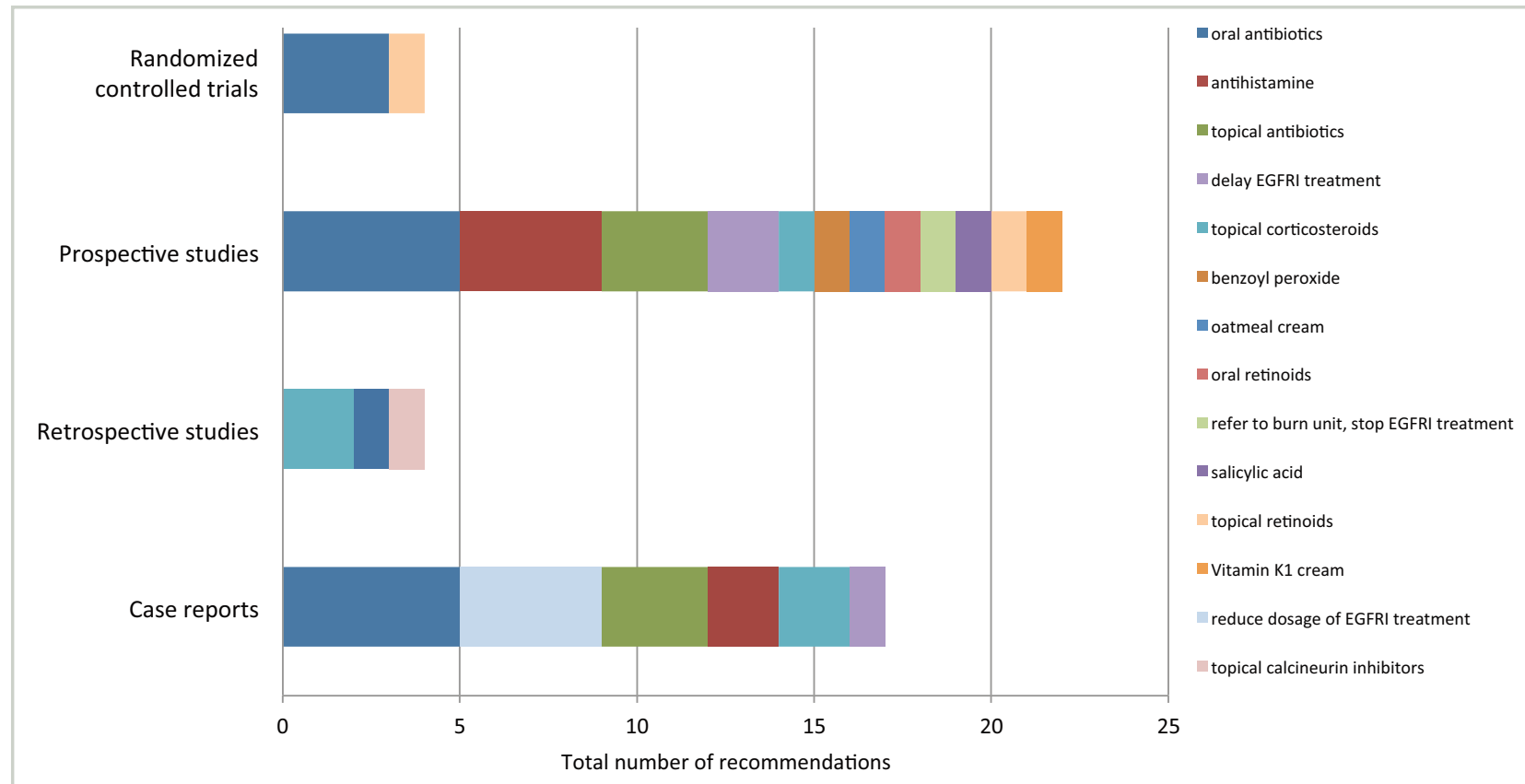

FIGURE 2 Rash management interventions by primary data source (excluding review articles, surveys, and clinical guidelines; $\mathrm{n}=20$ articles.) Counts indicate the number of articles that recommend a particular rash management intervention. A single article may have multiple recommendations.

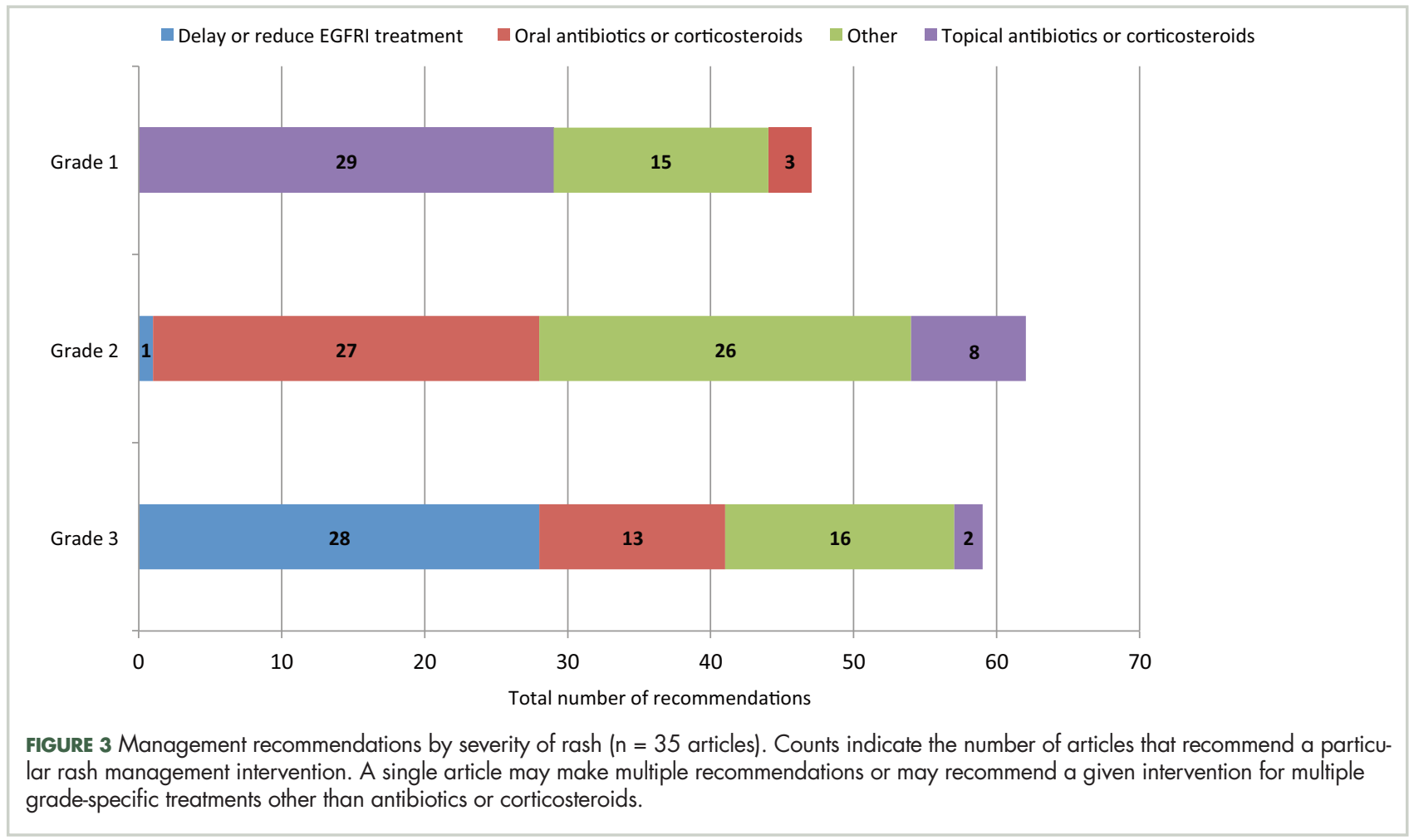

ing exclusion criteria, a total of 59 articles were selected. Articles published between 2005 and 2011 providing rash management recommendations were included in the review (Figure 1). None of the review articles were sys- tematic reviews. The articles identify a total of 20 different types of rash management treatments (Table 2). Most of the articles were from Europe (18) and the United States (34). 
TABLE 1 Classification of articles identified in the systematic review

\section{Article type}

Review article

Clinical practice guideline

Case report

Prospective study (non-RCT)

Retrospective study ${ }^{a}$

$\mathrm{RCTa}$

Cross-sectional survey ${ }^{b}$

$\mathrm{RCT}$, randomized-controlled trial

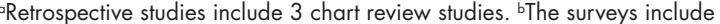
2 cross-sectional surveys of oncologists or other health care practitioners involved in rash management.

\section{Rash management interventions}

Regardless of grade of rash, the most commonly identified drug interventions within the selected publications include oral antibiotics, topical antibiotics, topical corticosteroids, and antihistamines (Table 2). Less common recommendations include oral corticosteroids, topical calcineurin inhibitors, oral retinoids, topical retinoids, benzoyl peroxide, anti-agents, and salicylic acid. Changes to EGFRI treatment are frequently recommended for severe rash, including delay, stopping treatment, or dose reduction.

A range of evidence sources were cited for rash management recommendations given in the 59 articles. Expert opinion was the most common evidentiary basis for recommendations. In addition, various primary research studies were identified, including RCTs, other interventional, prospective studies without a comparator arm, case report studies, and other retrospective studies. Figure 2 presents rash management intervention recommended by study design for the subset of primary research articles identified in the literature search.

As highlighted above, most of the articles identified in our search recommended both topical and oral antibiotic treatments. All $3 \mathrm{RCTs}^{31-33}$ and 5 of the 7 studies with other prospective designs supported their use. ${ }^{34-38}$

Topical corticosteroids were recommended as a rash management intervention in 24 of the 59 articles identified in this review, however, relatively little clinical evidence was identified to supports this recommendation. Topical and oral retinoids are also recommended for rash management (Table 2), although they are less often recommended than antibiotics or corticosteroids. ${ }^{39,40,33,35}$ Other, more unconventional treatments were also identified in the literature. Benzoyl peroxide was recommended based on 1 prospective study ${ }^{35}$ and 1 review article citing anecdotal reports, ${ }^{41}$ but specifically not recommended in 2 recent reviews. ${ }^{2,3}$
Topical wound gel decreased the itching sensation in a prospective study of 13 patients with a grade 2 rash. ${ }^{42}$ Vitamin K cream allowed $69.2 \%$ of patients good skin-rash symptom control in an uncontrolled prospective study. ${ }^{34} \mathrm{In}$ another uncontrolled prospective study, 43 treatment with topical oatmeal cream was associated with full-rash resolution in $64.3 \%$ of patients, partial improvement in $35.7 \%$, and overall response in $100 \%$ of patients who applied colloidal oatmeal lotion 3 times a day for 21 days.

\section{Reactive management by severity of rash}

Most of the 59 identified articles provide EGFRIassociated rash management strategies based on the severity of rash, including 27 of 37 review articles or clinical guidelines. Table 2 summarizes all recommendations for use of specific rash management interventions by grade of rash. These data illustrate a pattern of escalating rash management interventions by severity of rash: topical treatments are mostly recommended for grade 1 rash, oral treatments are mostly recommended for more severe rash, and delay or dose reduction of EGFRI are almost exclusively recommended for grade 3 rash (Figure 3).

\section{Pre-emptive rash management}

The timing of treatment may be important for effective rash management. Three RCTs evaluated preventing the onset of EGFRI-associated rash by using oral antibiotics prior to the onset of rash. ${ }^{31-33}$ All three RCTs found that preemptive oral antibiotics were well tolerated and showed signs of reducing severe skin toxicities, however, future studies are necessary to conclude whether this strategy prevents EGFRI rash. Only 1 of these RCTs compared the use of pre-emptive and reactive treatments. ${ }^{31}$ All 3 studies demonstrated that pre-emptive antibiotic treatment may decrease severity or incidence of rash, ${ }^{31,33}$ although 1 did not reach statistical significance. ${ }^{33}$

All of the other primary research articles focused on reactive rash management. ${ }^{34-38,42-43}$ Review articles and clinical guidelines often recommend early intervention to prevent worsening of rash, but while some guidelines strongly recommend pre-emptive use of oral antibiotics, ${ }^{3}$ other recent articles either do not discuss this approach ${ }^{44,45}$ or review the literature, but do not recommend this management strategy based on the available evidence., ${ }^{2,46}$

\section{Variation among management recommendations}

Despite a general trend by severity emerging in the rash management recommendations, Table 2 shows that substantial variation in these recommendations does exist within each grade of rash. Recommended treatments are also not always restricted to a grade of rash. Oral antibiotics are, for example, the most commonly recommended rash management intervention for grade 2 rash, but also are recommended for grade 1 and grade 3 rash, and a total of 
TABLE 2 Recommendations for managing EGFRl-associated rash based on grade ( $\mathrm{n}=59$ articles) ${ }^{a}$

\section{Rash grade}

\begin{tabular}{|c|c|c|c|c|c|c|c|}
\hline $\begin{array}{l}\text { Rash management } \\
\text { intervention }\end{array}$ & $\mathbf{I}$ & II & III & IV & All & Unspecified & Total \\
\hline Oral antibiotics & 4 & 23 & 2 & & 3 & 13 & 45 \\
\hline Topical corticosteroids & 8 & 4 & 1 & & 1 & 12 & 26 \\
\hline Delay EGFRI treatment & & & 17 & 1 & & 1 & 19 \\
\hline Oral corticosteroids & & 3 & 8 & 2 & & 2 & 15 \\
\hline $\begin{array}{l}\text { Reduce dosage of EGFR } \\
\text { treatment }\end{array}$ & & 1 & 8 & & & 4 & 13 \\
\hline
\end{tabular}

Refer to burn unit, stop

EGFRI treatment

8

8

\begin{tabular}{|c|c|c|c|c|c|c|}
\hline Topical retinoids & 1 & 2 & 1 & & 2 & 6 \\
\hline Benzoyl peroxide & 4 & 1 & & & 2 & 7 \\
\hline Compresses & & 1 & 5 & & 1 & 7 \\
\hline Stop EGFRI treatment & & & 2 & 4 & & 6 \\
\hline Anti-rosacea agents & 4 & & & & & 4 \\
\hline Oatmeal cream & 2 & & & & 2 & 4 \\
\hline Salicylic acid & 2 & 1 & & & 1 & 4 \\
\hline Vitamin K cream & & 2 & & & & 2 \\
\hline Drying pastes & & & 1 & & & 1 \\
\hline Hydrophilic dressings & & & 1 & & & 1 \\
\hline
\end{tabular}

EGFRI, epidermal growth factor receptor inhibitor

aCounts indicate the number of articles that recommend a particular rash management intervention. A single article may make multiple recommendations or may recommend a given intervention for multiple grades of rash.

16 articles recommend use of oral antibiotics unspecified by any particular grade (Table 2 ).

Some variation was observed in recommendations by article type. Seven of the 18 clinical practice guidelines were self-identified as consensus guidelines, incorporating recommendations from multidisciplinary teams that include radiation oncologists, medical oncologists, pharmacologists, and dermatologists. ${ }^{2,45,47-49}$ Although the consensus guidelines' recommendation to use oral treatments for higher grades of rash is consistent with other article types, consensus guidelines were the only article type that recommended oral steroids more frequently than oral anti- biotics across all grades of rash.

There was substantial variation in the terminology used to describe EGFRI-associated rash, which was cited in several articles as a potential source of variation in treatments recommended by grade of rash. ${ }^{49-53}$ The Common Terminology Criteria for Adverse Events (CTCAE) is frequently used within the context of phase 2 and 3 clinical trials investigating the efficacy of EGFRI therapy. Even when the CTCAE system is used, different rash descriptions can be reported; for example, separate descriptions exist for rash, skin rash, acneiform, acne-like rash, skin toxicity. ${ }^{51}$ Also variations in the rash grading system can 
be found between different versions of the CTCAE. ${ }^{54,55}$ The Medical Dictionary for Regulatory Activities ${ }^{8,13-14,56-58}$ is also often used to classify the grade of rash as well as other systems such as the Coding Symbols for a Thesaurus of Adverse Reaction Terms (COSTART). ${ }^{15}$ Notably, the severity and outcomes of rash management interventions typically relied on a physician's assessment.

\section{Discussion}

The objective of this systematic review was to identify and summarize all available published recommendations of rash management strategies and evaluate their basis of evidence. Fifty-nine articles were found, which included clinically relevant guidelines or opinions for treating EGFRIassociated rash. Most of the recommendations were based on expert opinion; consequently, quality scoring and metaanalysis of the data extracted from these articles were not conducted. Otherwise, PRISMA guidelines were followed for reporting this systematic review. ${ }^{30}$

Of the 19 review articles identified by our search, none were systematic reviews. The reviews did not comprehensively describe the existing literature nor did they summarize or analyze the trends in management recommendations described in the literature. Eighteen clinical practice guidelines for the effective management of EGFRI-associated rash were also identified. The focus of the guidelines was on the reactive management of EGFRI-associated rash.

The 59 articles, identified by the systematic search, presented a range of management recommendations. The most commonly recommended drug interventions were oral antibiotics, followed by topical antibiotics, topical corticosteroids and antihistamines. Although there was variation in treatments recommended for each grade of rash, a general pattern of rash management recommendation by grade did emerge, however the lack of consistency in grading may impact these results It was generally recommended that a low grade of rash is managed with topical antibiotics or corticosteroids, grade 2 rash is managed with oral antibiotics or antihistamines, and more severe grades of rash are managed with oral corticosteroids, and delay or dose reduction of EGFRI. EGFRI product labeling is consistent with recommendations for dose modification and therapy interruption for severe rash. ${ }^{26-29}$

Evidence supporting the use of oral corticosteroids is more limited. None of the identified prospective studies evaluated the effectiveness of oral corticosteroids in managing EGFRI-associated rash. Oral corticosteroids carry risks, and can increase the risk for infection for many patients ${ }^{3}$ and could potentially interfere with the activity of the 2 EGFRI treatments, cetuximab and panitumumab. ${ }^{48,50}$

With the exception of the pre-emptive strategies using oral antibiotics and topical corticosteroids and retinoids, the efficacy and tolerability of these management strategies have not been evaluated in the context of a con- trolled trial. Only 3 RCTs were identified by our systematic review. ${ }^{31-33}$ We may not have identified all the RCTs evaluating rash management programs as the focus of our search strategy was not randomized controlled studies of rash management options, rather it was limited to clinically relevant guidelines or opinions for treating EGFRIassociated rash. Nonetheless, the finding that there is a paucity of RCT-based evidence is consistent with the comments found in the literature. ${ }^{3,}$ The focus of the RCTs was on pre-emptive management of EGFRI-associated rash. One of the 3 trials compared pre-emptive management with reactive management, and suggested that preemptive treatment is much more effective than reactive treatment at reducing the incidence of grade 2 or higher rash. ${ }^{31}$ With limited information and a lack of phase 3 data, a better understanding of the effectiveness of preemptive versus reactive strategies with the implementation of appropriately designed RCTs could support a more effective management of EGFRI-associated rash and potentially improve patient outcomes.

One important outcome to consider in the design of future trials is patients' self-reported outcomes, such as symptoms or health-related quality of life. While the physical appearance of rash in terms of incidence and severity is a relevant outcome, the clinician's interpretation of the implications for the patient is not necessarily a good reflection of how the patient feels or perceives the severity of rash. A more consistent and precise measurement of the manifestations of rash incorporated in the design of future clinical trials would also help support the selection of alternative rash management strategies.

There is some debate and speculation over the presence of rash being associated with the efficacy of EGFRI therapy. ${ }^{51,52}$ If this association was to be confirmed with further research, the need for a better understanding of the effectiveness of reactive versus pre-emptive EGFRI-associated rash management strategies would nonetheless remain. Moreover, it would be of interest to investigate whether effective rash management affects the efficacy of EGFRI therapy.

A possible limitation of this review results from the inclusion of recommendations from review articles and guidelines as well as clinical studies. As such, it did not systematically search for primary research studies and may have omitted some relevant studies. Although the classification of study type is mutually exclusive there is some overlap in the studies cited by the review articles and guidelines and the primary research articles. The guidelines, however, relied mainly on expert opinion and there was generally a paucity of primary research data. In addition, some general, typically preventative, nonprescription interventions (“tips”) were also not specifically captured, including limiting sun exposure, using sunscreen, avoiding skin irritants, and so on. ${ }^{35,54,59}$ Nonetheless, we believe that the key conclusions of this analysis are robust to these limitations. 


\section{Conclusions}

Most management recommendations relied on expert opinion. Although there was variation in recommendations, it was generally recommended that low grade of rash is managed with topical antibiotics or corticosteroids, grade 2 rash with oral antibiotics or antihistamines, and more severe grades of rash with oral corticosteroids, and delay or dose reduction of EGFRI. A better understanding of pre-emptive versus reactive treatment with the implementation of appropriately designed randomized con-

\section{References}

1. Lacouture ME, Maitland ML, Segaert S, et al. A proposed EGFR inhibitor dermatologic adverse event-specific grading scale from the MASCC skin toxicity study group. Support Care Cancer. 2010;18:509-522.

2. Potthoff K, Hofheinz R, Hassel JC, et al. Interdisciplinary management of EGFR-inhibitor-induced skin reactions: a German expert opinion. Ann Oncol. 2011;22:524-535.

3. Lacouture M, Anadkat M, Bensadoun RJ, et al. MASCC Skin Toxicity Study Group. Clinical practice guidelines for the prevention and treatment of EGFR inhibitor-associated dermatologic toxicities. Support Care Cancer. 2011;19:1079-1095.

4. Shepherd FA, Rodrigues Pereira J, Ciuleanu T, et al. National Cancer Institute of Canada Clinical Trials Group. Erlotinib in previously treated non-small-cell lung cancer. N Engl J Med. 2005;353:123-132.

5. Moore MJ, Goldstein D, Hamm J, et al. National Cancer Institute of Canada Clinical Trials Group. Erlotinib plus gemcitabine compared with gemcitabine alone in patients with advanced pancreatic cancer: a phase III trial of the National Cancer Institute of Canada Clinical Trials Group. J Clin Oncol. 2007;25:1960-1966.

6. Kelly K, Chansky K, Gaspar LE, et al. Phase III trial of maintenance gefitinib or placebo after concurrent chemoradiotherapy and docetaxel consolidation in inoperable stage III non-small-cell lung cancer: SWOG S0023. J Clin Oncol. 2008;26:2450-2456.

7. Peeters M, Siena S, Van Cutsem E et al. Association of progressionfree survival, overall survival, and patient-reported outcomes by skin toxicity and KRAS status in patients receiving panitumumab monotherapy. Cancer. 2009;115:1544-1554.

8. Pirker R, Pereira JR, Szczesna A et al. Cetuximab plus chemotherapy in patients with advanced non-small-cell lung cancer (FLEX): an open-label randomised phase III trial. Lancet. 2009;373:1525-1531.

9. Tol J, Koopman M, Cats A et al. Chemotherapy, bevacizumab, and cetuximab in metastatic colorectal cancer. N Engl J Med. 2009;360:563-572.

10. Wagner LI, Lacouture ME. Dermatologic toxicities associated with EGFR inhibitors: the clinical psychologist's perspective. Impact on health-related quality of life and implications for clinical management of psychological sequelae. Oncol (Williston Park). 2007;21:34-36.

11. Jonker DJ, O'Callaghan CJ, Karapetis CS, et al. Cetuximab for the treatment of colorectal cancer. N Engl J Med. 2007;357:2040-2048.

12. Cascinu S, Berardi R, Labianca R, et al. Italian Group for the Study of Digestive Tract Cancer (GISCAD). Cetuximab plus gemcitabine and cisplatin compared with gemcitabine and cisplatin alone in patients with advanced pancreatic cancer: a randomised, multicentre, phase II trial. Lancet Oncol. 2008;9:39-44.

13. Okines AF, Ashley SE, Cunningham D, et al. Epirubicin, oxaliplatin, and capecitabine with or without panitumumab for advanced esophagogastric cancer: dose-finding study for the prospective multicenter, randomized, phase II/III REAL-3 trial. J Clin Oncol. 2010;28:3945-3950.

14. Bokemeyer C, Bondarenko I, A. Makhson A, et al. Fluorouracil, leucovorin, and oxaliplatin with and without cetuximab in the first-line treatment of metastatic colorectal cancer. J Clin Oncol. 2009;27:663-671. trolled studies could support a more effective management of EGFRI-associated rash and improve patient outcomes. A consistent grading of rash toxicity is necessary to accurately assess the severity of rash and guide the development of rash management strategies.

\section{Acknowledgments}

The authors thank Patricia Marinello, Bristol-Myers Squibb, for her comments on the manuscript, and Kimberly Yee and Jordan Rooklyn for help with data analysis and preparation of the manuscript.

15. Bonner JA, Harari PM, Giralt J et al. Radiotherapy plus cetuximab for squamous-cell carcinoma of the head and neck. N Engl J Med. 2006;354:567-578.

16. Borner M, Koeberle D, Von MR, et al. Swiss Group for Clinical Cancer Research (SAKK), Bern, Switzerland. Adding cetuximab to capecitabine plus oxaliplatin (XELOX) in first-line treatment of metastatic colorectal cancer: a randomized phase II trial of the Swiss Group for Clinical Cancer Research SAKK. Ann Oncol. 2008;19:1288-1292.

17. Bukowski RM, Kabbinavar FF, Figlin RA et al. Randomized phase II study of erlotinib combined with bevacizumab compared with bevacizumab alone in metastatic renal cell cancer. J Clin Oncol. 2007;25:4536-4541.

18. Butts CA, Bodkin D, Middleman EL, et al. Randomized phase II study of gemcitabine plus cisplatin or carboplatin [corrected], with or without cetuximab, as first-line therapy for patients with advanced or metastatic non small-cell lung cancer. J Clin Oncol. 2007;25:5777-5784.

19. Cristofanilli M, Valero V, Mangalik A, et al. Phase II, randomized trial to compare anastrozole combined with gefitinib or placebo in postmenopausal women with hormone receptor-positive metastatic breast cancer. Clin Cancer Res. 2010;16:1904-1914.

20. Gatzemeier U, Pluzanska A, Szczesna A et al. Phase III study of erlotinib in combination with cisplatin and gemcitabine in advanced non-small-cell lung cancer: the Tarceva Lung Cancer Investigation Trial. J Clin Oncol. 2007;25:1545-1552.

21. Guarneri V, Frassoldati A, Ficarra G, et al. Phase II, randomized trial of preoperative epirubicin-paclitaxel +/- gefitinib with biomarker evaluation in operable breast cancer. Breast Cancer Res Treat. 2008;110:127-134.

22. Lynch TJ, Patel T, Dreisbach L et al. Cetuximab and first-line taxane/carboplatin chemotherapy in advanced non-small-cell lung cancer: results of the randomized multicenter phase III trial BMS099. J Clin Oncol. 2010;28:911-917.

23. Mok TS, Wu YL, Yu CJ, et al. Randomized, placebo-controlled, phase II study of sequential erlotinib and chemotherapy as first-line treatment for advanced non-small-cell lung cancer. J Clin Oncol. 2009;27:5080-5087.

24. Tsuboi M, Kato H, Nagai K et al. Gefitinib in the adjuvant setting: safety results from a phase III study in patients with completely resected non-small-cell lung cancer. Anticancer Drugs. 2005; $16: 1123-1128$.

25. Van Cutsem E, Peeters M, Siena S, et al. Open-label phase III trial of panitumumab plus best supportive care compared with best supportive care alone in patients with chemotherapy-refractory metastatic colorectal cancer. J Clin Oncol. 2007;25:1658-1664.

26. Iressa [package insert]. Wilmington, DE: AstraZeneca Pharmaceuticals LP; 2004.

27. Tarceva [package insert]. Farmingdale, NY: OSI Pharmaceuticals, LLC; 2012.

28. Erbitux [package insert]. Branchburg, NJ: ImClone LLC; 2013.

29. Vectibix [package insert]. Thousand Oaks, CA: Amgen Inc; 2013.

30. Moher D, Liberati A, Tetzlaff J, Altman DG; PRISMA Group. Preferred reporting items for systematic reviews and meta-analyses: the PRISMA statement. BMJ. 2009;339:b2535. 
31. Lacouture ME, Mitchell EP, Piperdi B, et al. Skin toxicity evaluation protocol with panitumumab (STEPP), a phase II, open-label, randomized trial evaluating the impact of a pre-emptive skin treatment regimen on skin toxicities and quality of life in patients with metastatic colorectal cancer. J Clin Oncol. 2010;28:1351-1357.

32. Jatoi A, Rowland K, Sloan JA, et al. Tetracycline to prevent epidermal growth factor receptor inhibitor-induced skin rashes: results of a placebo-controlled trial from the North Central Cancer Treatment Group (N03CB). Cancer. 2008;113:847-853.

33. Scope A, Agero AL, Dusza SW, et al. Randomized doubleblind trial of prophylactic oral minocycline and topical tazarotene for cetuximab-associated acne-like eruption. J Clin Oncol. 2007;25:5390-5396.

34. Pinto C, Barone C, Martoni A, et al. Vitamin K1 cream in the management of skin rash during anti-EGFR monoclonal antibody $(\mathrm{mAb})$ treatment in patients with metastatic cancer: First analysis of an observational Italian study. J Clin Oncol. 2011;29:S594.

35. de Noronha e Menezes NM, Lima R, Moreira A, et al. Description and management of cutaneous side effects during erlotinib and cetuximab treatment in lung and colorectal cancer patients: a prospective and descriptive study of 19 patients. Eur J Dermatol. 2009:19:248-251.

36. Manganoni AM, Farisoglio C, Ferrari V, et al. Multidisciplinary team-working indicators of good practice in the clinical management of EGFR-inhibitor dermatologic toxicities. Ann Surg Oncol. 2009;16:224-225.

37. Racca P, Fanchini L, Caliendo V, et al. Efficacy and skin toxicity management with cetuximab in metastatic colorectal cancer: outcomes from an oncologic/dermatologic cooperation. Clin Colorectal Cancer. 2008;7:48-54.

38. Roé E, Garcia Muret MP, Marcuello E, Capdevila J, Pallarés C, Alomar A. Description and management of cutaneous side effects during cetuximab or erlotinib treatments: a prospective study of 30 patients. J Am Acad Dermatol. 2006;55:429-437.

39. Adams DH, Nutt T. A case report and discussion of cetuximabinduced folliculitis. Am J Clin Dermatol. 2006;7:333-336.

40. Saif MW, Kim R. Incidence and management of cutaneous toxicitie associated with cetuximab. Expert Opin Drug Saf. 2007;6:175-182.

41. Duvic M. EGFR inhibitor-associated acneiform folliculitis: assessment and management. Am J Clin Dermatol. 2008;9:285-294.

42. Wong SF, Lindgren A, Mummaneni M, et al. A prospective cross- over pilot study to evaluate the use of a topical wound gel in patients with cutaneous toxicity caused by epidermal growth factor receptor inhibitors. J Support Oncol. 2010;8:202-208.

43. Alexandrescu DT, Kauffman LC and Dasanu CA. Treatment of epidermal growth factor-induced rash with colloidal oatmeal extract: An extended treatment follow-up. J Invest Derm. 2009;129(Suppl. 1s):346.

44. Eng C. Toxic effects and their management: daily clinical challenges in the treatment of colorectal cancer. Nat Rev Clin Oncol. 2009;6:207-218.

45. Pinto C, Barone CA, Girolomoni G, et al. European Society of Medical Oncology. Management of skin toxicity associated with cetuximab treatment in combination with chemotherapy or radiotherapy. Oncologist. 2011;16:228-238.

46. Ouwerkerk J, Boers-Doets C. Best practices in the management of toxicities related to anti-EGFR agents for metastatic colorectal cancer. Eur J Oncol Nurs. 2010;14:337-349.

47. Burtness B, Anadkat, M, Basti, S, et al. NCCN Task Force Report: Management of dermatologic and other toxicities associated with EGFR inhibition in patients with cancer. J Natl Compr Canc Netw. 2009; 7:S5-S21; quiz S22-S24.

48. Sapadin AN, Fleischmajer R. Tetracyclines: nonantibiotic properties and their clinical implications. J Am Acad Dermatol. 2006;54:258-265.

49. Thatcher N, Nicolson M, Groves RW, et al. Expert consensus on the management of erlotinib-associated cutaneous toxicity in the UK. Oncologist. 2009;14:840-847.

50. Segaert S, Van Cutsem E. Clinical signs, pathophysiology and management of skin toxicity during therapy with epidermal growth factor receptor inhibitors. Ann Oncol. 2005;16:1425-1433.

51. Lynch TJ, Kim ES, Eaby B, Garey J, West DP, Lacouture ME. Epidermal growth factor receptor inhibitor-associated cutaneous toxicities: an evolving paradigm in clinical management. Oncologist. 2007;12:610-621.

52. Chou LS, Garey J, Oishi K, Kim E. Managing dermatologic toxicities of epidermal growth factor receptor inhibitors. Clin Lung Cancer. 2006;8:S15-S22.

53. Rhee J, Oishi K, Garey J, Kim E. Management of rash and other toxicities in patients treated with epidermal growth factor receptor-targeted agents. Clin Colorectal Cancer. 2005;5:S101-S106. 